\title{
MEASURING EMITTANCE GROWTH DUE TO MAGNETIC BUNCHING OF AN ELECTRON BEAM USING THE SECOND MOMENT OF ITS IMAGE CHARGE
}

\author{
$\underline{\text { S. J. Russell}}^{*}$ and B. E. Carlsten, Los Alamos National Laboratory
}

\begin{abstract}
Current thinking about the Next Linear Collider (NLC) places a great deal of importance on magnetic beam compression. However, theoretical work has predicted that a large emittance growth may occur during magnetic compression due to coherent synchrotron radiation and the non-inertial space-charge force[1]. Applications such as the NLC cannot tolerate such beam degradation. To verify this prediction we have measured the emittance of the Sub-Picosecond Accelerator electron beam as a function of compression in our magnetic chicane and compared the results to numerical simulation. These emittance measurements were carried out using a pair of quadrupole magnets and a beam position monitor.
\end{abstract}

\section{INTRODUCTION}

The Sub-Picosecond Accelerator facility (SPA) at Los Alamos National Laboratory is an $8 \mathrm{MeV}$, radiofrequency photoinjector operating at $1300 \mathrm{MHz}$ [2]. The primary mission of SPA is to explore the uses and dynamics of bunched electron beams. State of the art in its field, SPA has compressed electron pulses containing 1 $\mathrm{nC}$ of charge to sub-picosecond lengths[3].

Beam compression on the SPA is accomplished with a magnetic chicane, illustrated in Fig. 1. To first order this series of dipole magnets is achromatic and has no net effect on the quality of the electron beam. However, recent work has identified two space-charge induced forces for beams in circular motion that are mostly independent of energy. In contrast, space-charge forces in straight-line motion scale inversely with the relativistic factor $\gamma$.

The first of these effects is considered a space-charge curvature effect and is known as the noninertial spacecharge force, in which the energies of the particles are modified with little total loss by radiation. The second effect is known as the coherent synchrotron radiation force, in which the bunch radiates coherently. Both effects will lead to a redistribution of the energy of a bunch in circular motion within an achromatic bend. In turn, this redistribution can lead to an unacceptable increase in the beam's bend plane emittance that would be roughly independent of beam energy.

"Email: srussell@lanl.gov
We have investigated this predicted emittance growth both numerically and experimentally. The experimental emittance values were obtained using Roger Miller's technique that measures the second moment of the beam image charge[4]. The current results of our investigations are presented here.

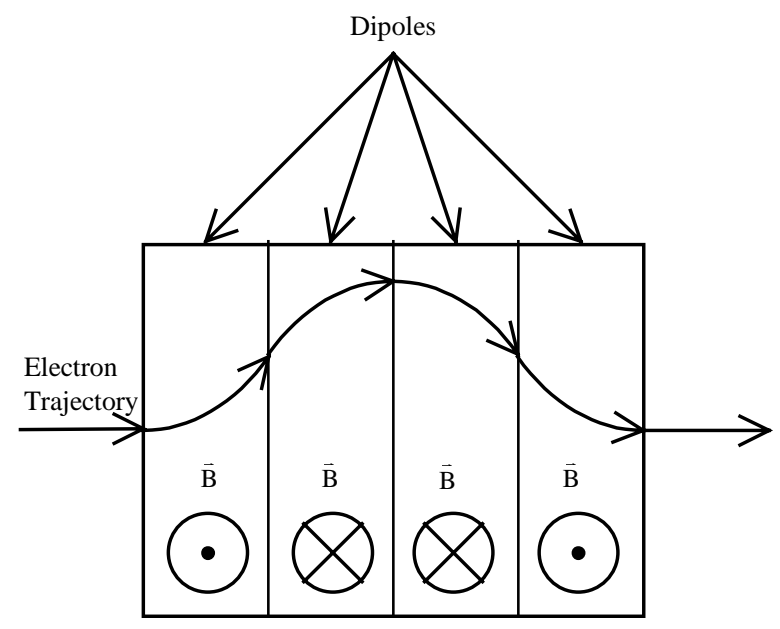

Figure 1: Motion of electron through magnetic chicane.

\section{EXPERIMENT}

Figure 2 is a schematic of the diagnostic section of the SPA beam line. The electron beam from the photoinjector enters from the left side of the diagram and is bunched by the chicane. The emittance is then measured utilizing the second two quadrupole magnets and the first beam position monitor (BPM)[4].

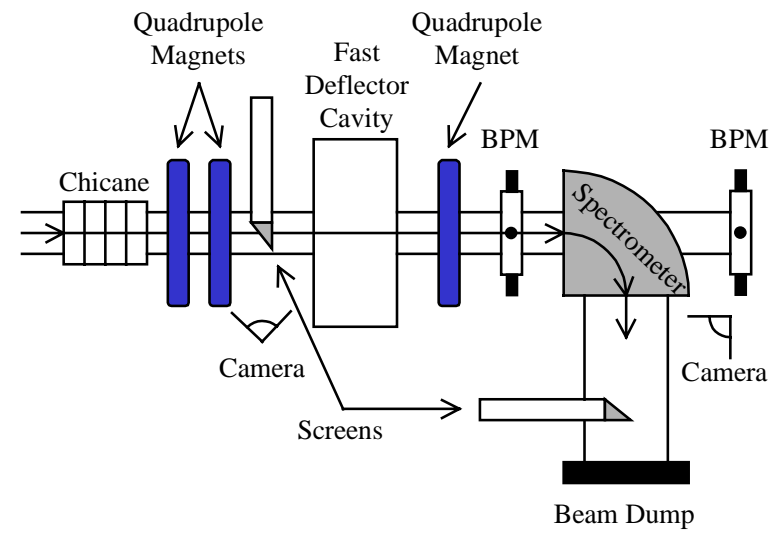

Figure 2: Schematic of experimental section of beam line. 


\subsection{Beam Bunching}

By correctly timing the arrival of the photoinjector drive laser pulse with respect to the rf fields in the accelerator, an energy versus phase correlation is generated across the beam bunch. Figure 3 shows an example from a PARMELA[5] simulation. Because the electrons at the front of the pulse have lower energy, they will travel a longer path through the chicane, Figure 1. If the angle of bend in the chicane, the energy versus phase correlation and initial length of the electron beam bunch are each chosen correctly, the beam bunch will be compressed to sub-picosecond lengths at the chicane exit.

Electron Energy vs. Position in Bunch

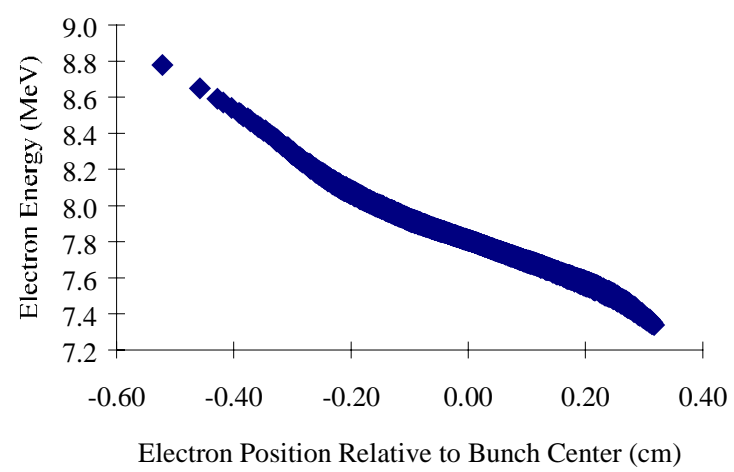

Figure 3: Energy versus electron position in beam bunch for efficient beam bunching from simulation.

\subsection{Emittance Measurement}

The emittance measurement technique used in these experiments was first suggested by Roger Miller et. al.[6] and later used to measure emittance on the SPA[4]. It employs two quadrupole magnets and a BPM. A cross section of the BPM is illustrated in Figure 4.

The normalized rms emittances are defined as

$$
\varepsilon_{\mathrm{xn}} \equiv \beta \gamma \sqrt{\left\langle\mathrm{x}^{2}\right\rangle\left\langle\mathrm{x}^{\prime 2}\right\rangle-\left\langle\mathrm{xx}^{\prime}\right\rangle^{2}} \text {, }
$$

and

$$
\varepsilon_{\mathrm{yn}} \equiv \beta \gamma \sqrt{\left\langle\mathrm{y}^{2}\right\rangle\left\langle\mathrm{y}^{\prime 2}\right\rangle-\left\langle\mathrm{yy}^{\prime}\right\rangle^{2}} .
$$

The angled brackets indicate an ensemble average over the beam's spatial distribution. To measure these quantities, the value of the vector

$$
\overrightarrow{\mathbf{x}} \equiv\left(\begin{array}{c}
\left\langle\mathrm{x}^{2}\right\rangle \\
\left\langle\mathrm{xx}^{\prime}\right\rangle \\
\left\langle\mathrm{x}^{\prime 2}\right\rangle \\
\left\langle\mathrm{y}^{2}\right\rangle \\
\left\langle\mathrm{yy}^{\prime}\right\rangle \\
\left\langle\mathrm{y}^{\prime 2}\right\rangle
\end{array}\right)
$$

must be determined at a single point in the beam line.

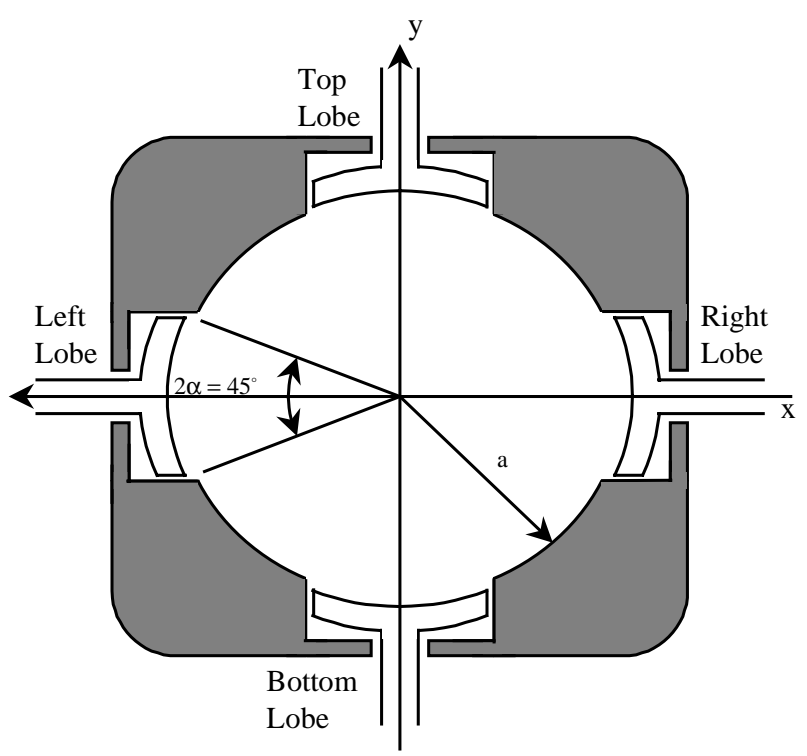

Figure 4: BPM schematic.

A BPM such as the one pictured in Figure 4 is capable of measuring not only the position of the beam center, $(\bar{x}, \bar{y})$, but also the second moment of the beam image charge:

$$
\left\langle\mathrm{x}^{2}\right\rangle-\left\langle\mathrm{y}^{2}\right\rangle+\bar{x}^{2}-\bar{y}^{2}=\mathrm{a}^{2} \frac{\alpha}{\sin 2 \alpha} \frac{\mathrm{A}_{\mathrm{R}}+\mathrm{A}_{\mathrm{L}}-\mathrm{A}_{\mathrm{T}}-\mathrm{A}_{\mathrm{B}}}{\mathrm{A}_{\mathrm{R}}+\mathrm{A}_{\mathrm{L}}+\mathrm{A}_{\mathrm{T}}+\mathrm{A}_{\mathrm{B}}} .
$$

$A_{R}, A_{L}, A_{T}$ and $A_{B}$ are the signal amplitudes of the right, left, top and bottom electrodes of the BPM. The accuracy of this measurement will be to order (beam size/BPM radius $)^{4}[6]$.

$$
\begin{aligned}
& \text { It can be shown that } \\
& \begin{aligned}
\left\langle x^{2}\right\rangle_{\mathrm{f}}- & \left\langle\mathrm{y}^{2}\right\rangle_{\mathrm{f}}=\left(\mathrm{R}_{11}\right)^{2}\left\langle\mathrm{x}^{2}\right\rangle_{\mathrm{i}}+2 \mathrm{R}_{11} \mathrm{R}_{12}\langle\mathrm{xx}\rangle_{\mathrm{i}}+\left(\mathrm{R}_{12}\right)^{2}\left\langle\mathrm{x}^{\prime 2}\right\rangle_{\mathrm{i}} \\
& -\left(\mathrm{R}_{33}\right)^{2}\left\langle\mathrm{y}^{2}\right\rangle_{\mathrm{i}}-2 \mathrm{R}_{33} \mathrm{R}_{34}\left\langle\mathrm{yy}^{\prime}\right\rangle_{\mathrm{i}}-\left(\mathrm{R}_{34}\right)^{2}\left\langle\mathrm{y}^{\prime 2}\right\rangle_{\mathrm{i}}[6],
\end{aligned}
\end{aligned}
$$

where the $f$ subscript refers to the BPM location and the $i$ subscript to a point upstream from the BPM. The constants $\mathrm{R}_{\mathrm{jk}}$ are from the transfer matrix for the focusing channel between the upstream point and the BPM:

$$
\overrightarrow{\overrightarrow{\mathbf{R}}}=\left[\begin{array}{cccc}
\mathrm{R}_{11} & \mathrm{R}_{12} & 0 & 0 \\
\mathrm{R}_{21} & \mathrm{R}_{22} & 0 & 0 \\
0 & 0 & \mathrm{R}_{33} & \mathrm{R}_{34} \\
0 & 0 & \mathrm{R}_{43} & \mathrm{R}_{44}
\end{array}\right] .
$$

Changing this transfer matrix $\mathrm{m}$ times $(\mathrm{m} \geq 6)$, and measuring

$$
\left\langle\mathrm{x}^{2}\right\rangle_{\mathrm{f}}-\left\langle\mathrm{y}^{2}\right\rangle_{\mathrm{f}}
$$

for each change, results in a matrix equation that can be solved for $\overrightarrow{\mathbf{x}}$ in the least squares sense. This estimates the rms beam parameters and, from (1) and (2), the normalized $\mathrm{x}$ and $\mathrm{y}$ emittances at the start of the focusing channel. 


\subsection{Experimental Apparatus}

In Figure 2, the quadrupoles are identical. They are electromagnetic, have a pole length of 2.75 inches and a gap radius of one inch. The fields of these quadrupoles have been simulated and measured. From this it has been determined that their effective length is $86 \mathrm{~mm}$ and that, at a radius of $1 \mathrm{~cm}$, the multipole components of the field are less than 1 percent of the quadrupole field. Attached to one pole of each magnet is a small Hall probe. The Hall probe voltages have been correlated to the gradients of the quadrupole fields. During beam operation, monitoring these voltages enables us to determine these gradients to within a percent.

The BPMs are dual axis, capacitive type probes that differentiate the beam image charge[7]. They were calibrated using a pulsed wire[8], [9].

The spectrometer is an electromagnetic dipole magnet that bends the beam $90^{\circ}$. Its edge angles are such that it focuses the beam on the screen shown in Figure 2. The spectrometer has been calibrated so that the average energy can be determined with two- percent accuracy.

The distance between the third quadrupole and the first BPM is $160 \mathrm{~mm}$. The distance between the second and third quadrupoles is $850 \mathrm{~mm}$. As stated before, these two quadrupoles and the first BPM are used to measure the emittance. This was accomplished by setting the two magnets to different field strengths and measuring the second moment of the beam with the BPM. These field strengths were carefully chosen to avoid unstable numerical conditions in the measurement[10].

A schematic of the data acquisition system used for capturing the signals from the BPM electrodes is shown in Figure 5. The signals from the four BPM electrodes travel down $50 \mathrm{Ohm}$, coaxial Heliax ${ }^{\mathrm{TM}}$ cables of equal length to $300 \mathrm{MHz}$, low-pass filters. From there they go to a Tektronix $^{\mathrm{TM}}$ TDS $684 \mathrm{C}$ digitizing oscilloscope. The oscilloscope digitizes each of its four channels at five giga-samples per second with eight bit accuracy. It is set to be bandwidth limited to $250 \mathrm{MHz}$. The oscilloscope is linked to a PC running LabView (C) via GPIB.

The electron beam consists of a single beam bunch with a rep rate of $1 \mathrm{~Hz}$. Upon capturing a beam shot, the 684C measures the peak-to-peak voltage of the BPM electrode signals and passes the information to the PC. The PC then calculates the beam intensity, position and second moment according to the BPM calibration. Typically when making emittance measurements, the parameters from 10 consecutive beam shots are averaged.

\section{EXPERIMENTAL RESULTS}

Two experiments were performed to investigate the predicated bend plane emittance growth. Each was done at a beam energy of $7.14 \mathrm{MeV}$ and a nominal beam bunch charge of $1 \mathrm{nC}$. In the first the emittance was measured versus chicane bend angle with the phase of the electron beam set to approximately $20^{\circ}$ with respect to the rf field zero. In the second the emittance is measured versus beam phase at a bend angle of $37^{\circ}$. In both experiments the maximum bunching was expected to occur at a phase of $20^{\circ}$ and a chicane bend angle of $37^{\circ}$.

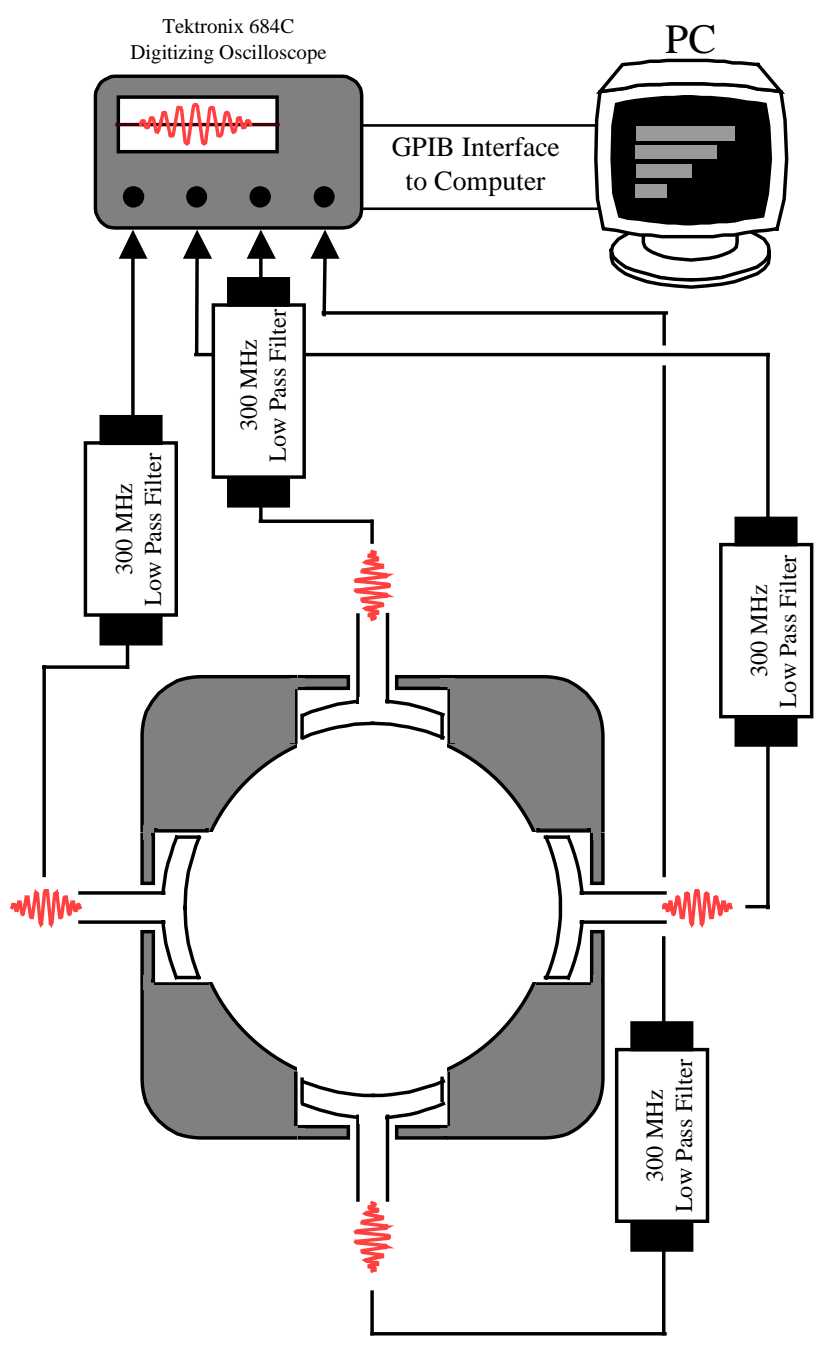

Figure 5: Schematic of data acquisition system.

It should be noted that during experiments the phase of the beam was not set with respect to the rf field zero. Instead the phase of the beam was first set so that the energy spread of the beam was a minimum. All other phase settings of the beam were then referenced to this point. This proved to be a much more practical, accurate, and reproducible reference point than the rf field zero. However, when comparing experimental results to numerical simulations of the SPA using PARMELA we needed to translate results to use the field zero to reference our phase. Since we have no good diagnostic for this, the experimental phases quoted here could be offset by several degrees.

At this time we do not have a bunch length diagnostic in place to measure the length of the electron beam 
directly. Instead we rely on the observation of beam energy spread as described in [2]. That is, the energy spread of the beam will dip just before maximum beam bunching and increase dramatically when maximum beam bunching is achieved. Although this is a very good qualitative, online diagnostic for tuning the beam, the actual bunch length must be inferred from this measurement. This is less than satisfying. Presently we are working to implement more quantitative measurements of the beam bunch length[11].

\subsection{Emittance Growth versus Bend Angle}

Figure 6 shows a plot of the experiment measuring the emittance of the electron beam in the bend plane (x plane for the SPA) versus the chicane bend angle. Also pictured are the results of PARMELA simulations in which we attempted to duplicate the experimental conditions. The maximum beam bunching occurs at an angle of $37^{\circ}$. The peak current of the beam at this point is expected to be approximately $800 \mathrm{~A}$, corresponding to a beam roughly 1.25 pico-seconds in length.

Normalized Emittance vs. Bend Angle

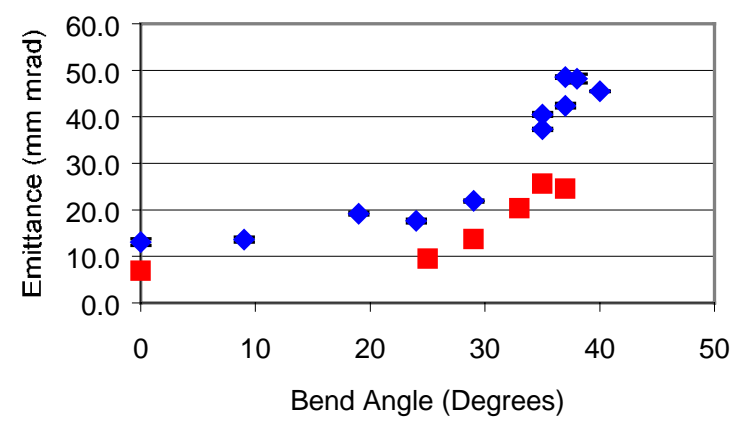

Measured Emittance $\square$ Calculated Emittance

Figure 6: Normalized rms emittance in the bending plane versus chicane bending angle. Results of experiment and numerical calculation.

Although the shapes of the emittance curves are similar, we see a much greater increase in the emittance experimentally than theory predicts. Assuming that the emittance increase adds in quadrature to the initial emittance, simulation predicts an emittance growth of 24.6 $\mathrm{mm}$ mrad. Experimentally, however, we observe an increase of $46.9 \mathrm{~mm}$ mrad.

\subsection{Emittance Growth versus Beam Phase}

Figure 7 shows a plot of the experiment measuring the emittance of the electron beam in the bend plane versus the beam phase at a chicane angle of $37^{\circ}$. This experiment was performed to verify that the emittance growth in the previous experiment cannot be attributed to poor beam transport through the chicane.
Normalized Emittance vs. Beam Phase

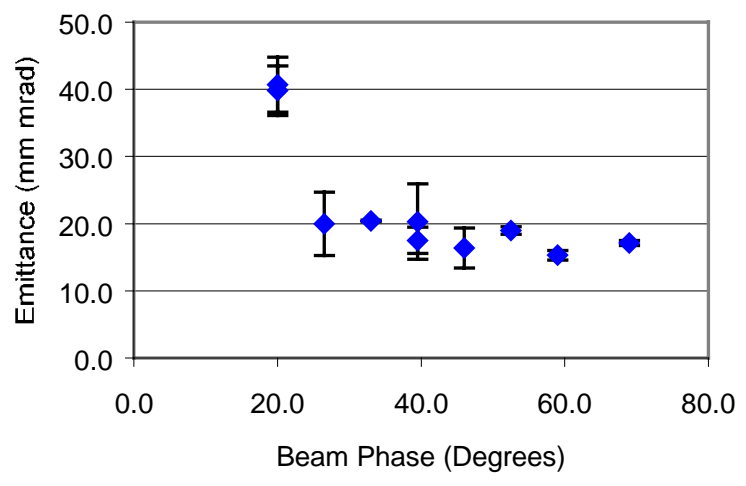

Figure 7: Normalized rms emittance in the bending plane versus beam phase at a chicane bending angle of $37^{\circ}$

What is important about this data set is that the emittance remains fairly constant until the phase reaches the maximum bunching phase of $20^{\circ}$. Then it jumps to about $40 \mathrm{~mm} \mathrm{mrad}$, very close to what was observed in the previous experiment. This seems to confirm poor beam transport is not the cause of the observed emittance growth in the first experiment.

\subsection{Discrepancy between Theory and Experiment}

The disagreement between theory and experiment is significant. We have identified two likely explanations. The first is a possible error in the calibration of our charge diagnostic. If the charge was higher than $1 \mathrm{nC}$ in these experiments, much of this discrepancy could be explained. The second possible explanation is a disagreement between the shape of the drive laser pulse in the experiment and the shape of the drive laser pulse assumed in the simulations. In the simulations, a Gaussian shape was used for the laser pulse. A recent, unrelated experiment has indicated that the real drive laser pulse is asymmetric with a long tail. We have not yet determined what effect, if any, this would have on our results.

\section{CONCLUSION}

We have successfully measured the emittance growth of an electron beam as it is bunched in a magnetic chicane due to the coherent synchrotron radiation and non-inertial space-charge force. However, there is a significant discrepancy between simulation and what was observed. We have identified two possible reasons for this discrepancy and hope to confirm or discredit each in the near future. 


\section{REFERENCES}

[1] B. E. Carlsten, "Calculation of the Noninertial Space-Charge Force and the Coherent Synchrotron Radiation Force for Short Electron Bunches in Circular Motion Using the Retard Green's Function Technique,” Phys. Rev. E 54, 838 (1996).

[2] Bruce E. Carlsten et. al., "Subpicosecond Compression Experiments at Los Alamos National Laboratory," Proceedings of the Micro Bunches Workshop, AIP Conf. Proc. 367, 21 (AIP, Upton, NY, 1995).

[3] B. E. Carlsten and S. J. Russell, "Subpicosecond compression of 0.1-1 nC electron bunches with a magnetic chicane at $8 \mathrm{MeV}$," Phys. Rev. E 53, 2072 (1996).

[4] Steven J. Russell, "Emittance Measurements of the Sub-picosecond Accelerator Electron Beam Using Beam Position Monitors," Rev. of Sci. Instrum. 70, 1362 (1999).

[5] L. M. Young, (private communication).

[6] R. H. Miller J. E. Clendenin, M. B. James, J. C. Sheppard, Proceedings of the $12^{\text {th }}$ International Conference on High Energy Accelerators, 602 (Fermilab, 1983).

[7] J. D. Gilpatrick, J. F. Power, R. E. Meyer and C. R. Rose, Proceedings of the 1993 Particle Accelerator Conference, IEEE catalog No. 93CH3279-7, 2334 (IEEE, New York, 1993).

[8] J. F. Power, J. D. Gilpatrick, F. Neri and R. B. Shurter, Proceedings of the 1992 Linear Accelerator Conference, AECL catalog No. 10728, 362 (AECL Research, Chalk River, Ontario, Canada, 1992).

[9] S. J. Russell, J. D. Gilpatrick, J. F. Power and R. B. Shurter, Proceedings of the 1995 Particle Accelerator Conference, IEEE catalog No. 95CH35843, 2580 (IEEE, New York, 1996).

[10] S. J. Russell, "Unstable Matrix Equations and Their Relationship to Measuring the Emittance of an Electron Beam Using Beam Position Monitors," (Accepted for publication in Nucl. Instrum. Phys. Res. A).

[11] S. J. Russell, "Measuring the RMS length of Short Electron Pulses with an RF cavity and a Beam Position Monitor," Proceedings of the 1997 Particle Accelerator Conference, IEEE catalog No. 97CB36167, 2005 (IEEE, New York, 1998). 